\title{
Torque Reversal in Accretion-Powered X-ray Pulsars
}

\author{
Insu $\mathrm{Yi}^{1}$, J. Craig Wheeler ${ }^{2}$, and Ethan T. Vishniac ${ }^{2}$ \\ ${ }^{1}$ Institute for Advanced Study, Princeton, NJ 08540; yi@sns.ias.edu \\ ${ }^{2}$ Astronomy Department, University of Texas, Austin, Texas 78712; ethan@grendel.as.utexas.edu, \\ wheel@astro.as.utexas.edu
}

\begin{abstract}
Accretion-powered X-ray pulsars 4U 1626-67, GX 1+4, and OAO 1657-415 have recently shown puzzling torque reversals. These reversals are characterized by short time scales, on the order of days, nearly identical spin-up and spin-down rates, and very small changes in X-ray luminosity. We propose that this phenomenon is the result of sudden dynamical changes in the accretion disks triggered by a gradual variation of mass accretion rates. These sudden torque reversals may occur at a critical accretion rate $\sim 10^{15}-10^{16} \mathrm{~g} \mathrm{~s}^{-1}$ when the system makes a transition from (to) a primarily Keplerian flow to (from) a substantially sub-Keplerian, radial advective flow in the inner disk. For systems near spin equilibrium, the spin-up torques in the Keplerian state are slightly larger than the spin-down torques in the advective state, in agreement with observation. The abrupt reversals could be a signature of pulsar systems near spin equilibrium with the mass accretion rates modulated on a time scale of a year or longer near the critical accretion rate. It is interesting that cataclysmic variables and black hole soft X-ray transients change their X-ray emission properties at accretion rates similar to the pulsars' critical rate. We speculate that the dynamical change in pulsar systems shares a common physical origin with white dwarf and black hole accretion disk systems.
\end{abstract}

Subject headings: accretion, accretion disks - stars: magnetic fields - stars: pulsars: general - X-rays: stars

\section{Introduction}

Detailed spin evolution on long time scales has been made available for several accretionpowered X-ray pulsars such as 4U 1626-67, GX 1+4, and OAO 1657-415 (for comprehensive reviews and data, see e.g. Chakrabarty et al. 1993, Chakrabarty 1995, and references therein). These systems have shown puzzling abrupt spin reversals. Before and after the observed torque reversals, their spin-up and spin-down torques were largely steady. It is intriguing that the spin-up 
and spin-down torques before and after reversals are nearly identical. These reversals are quite different from the random torque fluctuations seen in some pulsar systems believed to be fed by winds (e.g. Nagase 1989, Anzer \& Börner 1995, and references therein). The nearly steady torques plausibly indicate the existence of ordered accretion disks. The observations indicate that the mass accretion rate is gradually modulated with small amplitudes on a time scale of at least a year, which is much longer than the typical reversal time scale.

In the disk-magnetosphere interaction models of the Ghosh-Lamb type (Ghosh \& Lamb 1979ab, Campbell 1992, Yi 1995, Wang 1995), the magnetic torque is a function of the mass accretion rate, $\dot{M}$. The sign of the torque is reversed (spin-up/down) as $\dot{M}$ varies when the disk inner edge moves past the equilibrium radius at which the torque vanishes (e.g. Lipunov 1992). In this picture, however, the torque variation is expected to be smooth and continuous unless $\dot{M}$ varies discontinuously. Although this behavior may be relevant for some smooth torque reversals, the observed sudden reversals appear distinct (Chakrabarty 1995). Given the lack of any plausible mechanism for discontinuous change of $\dot{M}$, which must be tuned to occur near spin equilibrium, it is difficult for the existing magnetized disk models to provide an explanation. The observations indicate that the mass accretion rates vary little during transition (cf. Chakrabarty 1995), which makes the discontinuous change of $\dot{M}$ unlikely as an explanation.

In this Letter, we propose a possible explanation for the observed sudden torque reversal. We take the neutron star moment of inertia $I_{*}=10^{45} \mathrm{~g} \mathrm{~cm}^{2}$, radius $R_{*}=10^{6} \mathrm{~cm}$, and mass $M_{*}=1.4 M_{\odot}$. At a cylindrical radius $R$ from the star, the vertical component of the dipole magnetic field $B_{z}(R)=B_{*}\left(R_{*} / R\right)^{3}$ where $B_{*}$ is the stellar surface field strength. The spin period is $P_{*}$ or angular velocity $\Omega_{*}=2 \pi / P_{*}$.

\section{Keplerian Disk-Magnetosphere Interaction and Inner Region}

In the conventional disk-magnetosphere interaction model of the Ghosh-Lamb type (Ghosh \& Lamb 1979ab), the magnetic field of an accreting neutron star penetrates a geometrically thin accretion disk and exerts a magnetic torque. Except in a narrow region near the radius where the disk is disrupted, it is assumed that the accretion disk rotation is Keplerian, $\Omega_{K}(R)=\left(G M_{*} / R^{3}\right)^{1 / 2}$, the radial internal pressure gradient is small, the radial drift velocity is small, and the disk thickness becomes negligible (Campbell 1992, Yi 1995, Wang 1995 and references therein). In such a model, the $\phi$ component of the induction equation in steady state gives the azimuthal component of the field

$$
B_{\phi}(R)=\frac{\gamma}{\alpha} \frac{\Omega_{*}-\Omega_{K}(R)}{\Omega_{K}(R)} B_{z}(R) .
$$

where we have assumed that the internal viscosity and the magnetic diffusivity are due to a single turbulent process. Here we will assume that $\gamma$, defined as the ratio of $R$ to the vertical velocity 
shear length scale $\left|v_{\phi} /\left(\partial v_{\phi} / \partial z\right)\right|$, is $\sim 1$ (e.g. Campbell 1992, Yi 1995). The parameter $\alpha$ is the usual viscosity parameter (e.g. Frank et al. 1992) and we take $\alpha=0.3$. Although we adopt specific values of $\gamma$ and $\alpha$, the constraints on $B_{*}$ could always be rescaled in such a way that the exact individual values of the three parameters are not necessary (e.g. Kenyon et al. 1996). The inner edge of the disk, where the disk is magnetically disrupted, is at $R=R_{o}$ determined by the condition that the magnetic torque exceeds the internal torque in the disk (Campbell 1992, Yi 1995, Wang 1995) which can be expressed as

$$
\left(\frac{R_{o}}{R_{c}}\right)^{7 / 2}=\frac{2 N_{c}}{\dot{M}\left(G M_{*} R_{c}\right)^{1 / 2}}\left[1-\left(\frac{R_{o}}{R_{c}}\right)^{3 / 2}\right]
$$

where $N_{c}=(\gamma / \alpha) B_{c}^{2} R_{c}^{3}, B_{c}=B_{z}\left(R=R_{c}\right)$, and $R_{c}=\left(G M_{*} P_{*}^{2} / 4 \pi^{2}\right)^{1 / 3}$ is the Keplerian corotation radius. Integrating the magnetic torque over the disk, and allowing for the angular momentum carried by the gas which crosses the inner edge of the disk, the torque exerted on the star by the disk is

$$
N=\frac{7}{6} N_{0} \frac{1-(8 / 7)\left(R_{o} / R_{c}\right)^{3 / 2}}{1-\left(R_{o} / R_{c}\right)^{3 / 2}}
$$

where $N_{0}=\dot{M}\left(G M_{*} R_{o}\right)^{1 / 2}$ (Campbell 1992, Yi 1995, Wang 1995) and $N \rightarrow 0$ as $R_{o} / R_{c}$ approaches the equilibrium point $x_{e q}=0.915$. This estimate is based on the assumption that there is a negligible flux of angular momentum from $R<R_{o}$. Most of the contribution to the torque in this type of model comes from field-disk interaction in a narrow region just outside of $R_{o}$. Several different phenomenological descriptions of disk-magnetosphere interaction cause little practical differences to our conclusions (Wang 1995).

Fig. 1(a) presents an example of the typical smooth torque reversal expected in the models of the Ghosh-Lamb type is shown. This example is based on a set of parameters similar to what we adopt below for $4 \mathrm{U}$ 1626-67. It is clear that any densely sampled spin evolution would reveal a gradual and continuous variation of the torque, a generic prediction of the Ghosh-Lamb type model. A sudden torque reversal with nearly constant $|N|$ is hard to explain unless there exists an unknown constraining mechanism or the $\dot{M}$ variation is discontinuous and fine-tuned. It is possible that some observed smooth torque reversals (Chakrabarty 1995) could be due to this type of reversal model.

Under suitable conditions the inner parts of the accretion disk may evolve to an optically thin, low density state, for example, inside the disk boundary layer around a white dwarf (e.g. Paczynski 1991, Narayan and Popham 1993). When $\dot{M}$ is sufficiently small this transition may occur over a broad range of radii within a disk, producing a state in which radiative losses are so inefficient that the disk retains a large fraction of the heat generated from the dissipation of orbital energy (the 'advective state', cf. Narayan and Yi 1995). The transition to this state is not well understood, and may be affected by various external factors, including X-ray irradiation (e.g. Meyer \& Meyer-Hofmeister 1990) and coronal heating (Meyer \& Meyer-Hofmeister 1994).

Here we will assume that, whatever the details of the transition, the disk will make the jump 
to a low density state when it becomes possible for it to do so. What is the critical $\dot{M}_{\text {crit }}$ below which the disk becomes hot and optically thin? Observationally, weakly magnetized cataclysmic variables generally show a trend in which the X-ray to optical flux ratio decreases as $\dot{M}$ increases. Above a critical rate of $\sim 10^{16} \mathrm{~g} \mathrm{~s}^{-1}$, the X-ray emission becomes extremely weak. This has been attributed to the transition of the inner region to an optically thin (X-ray emitting) hot accretion disk (Patterson \& Raymond 1985, Narayan \& Popham 1993) at low $\dot{M}$. Interestingly, such a critical rate is largely consistent with the critical rate $\sim 2 \times 10^{17} \alpha^{2}$ or $\sim 2 \times 10^{16} \mathrm{~g} \mathrm{~s}^{-1}$ for $\alpha \sim 0.3$ based on the recently discussed advection-dominated hot accretion disks (Narayan \& Yi 1995). For a given accretion rate, there exists a critical radius inside of which the disk makes a transition to sub-Keplerian while the disk at radii larger than the critical radius remains Keplerian (Narayan \& Yi 1995). The exact location of this critical radius is not clearly understood yet. We assume that most of the magnetic torque contribution comes from the inner region (e.g. Wang 1995) which is inside the critical radius, e.g. $R_{\text {crit }} \gg R_{c}>R_{o}$. The relevance of the critical $\dot{M}_{\text {crit }}$ for the cataclysmic variables becomes more striking when we consider strongly magnetized neutron star systems. The inner edge of the accretion disk around a strongly magnetized neutron star lies roughly at $R_{o} \sim 5 \times 10^{8}\left(B_{*} / 10^{12} G\right)^{4 / 7}\left(\dot{M} / 10^{16} \mathrm{~g} / \mathrm{s}\right)^{-2 / 7} \mathrm{~cm}$ which is close to the typical white dwarf radius. Therefore, one may ask what would be the effects of the transition, at $\dot{M}_{c r i t} \sim 10^{16} \mathrm{~g} \mathrm{~s}^{-1}$, to the hot optically thin accretion disk on the spin evolution of the pulsars?

\section{Disk Transition and Torque Reversal}

We propose an explanation for the torque reversal based on such a transition. The optically thin, hot accretion disk cannot be geometrically thin or Keplerian once its internal pressure $\sim \rho c_{s}^{2}$ becomes a significant fraction of its orbital energy. After the transition, the disk thickness $H \sim c_{s} / \Omega_{K}$ and radial drift velocity $v_{R} \sim \alpha c_{s}^{2} / \Omega_{K}$ increase. The rotation of the accretion disk $\Omega$ becomes sub-Keplerian $\Omega<\Omega_{K}$ (e.g. Narayan \& Yi 1995). In the case of strongly magnetized pulsars, direct X-ray observation of such a disk transition is difficult because most of the X-ray luminosity, $L_{x} \sim G M_{*} \dot{M} / R_{*}$, comes from the surface of the star and the luminosity from the disk, which is truncated well above the stellar surface, is limited to $\sim G M_{*} \dot{M} / R_{o} \ll L_{x}$. The sub-Keplerian disk, however, may have observable dynamical consequences. Once the sub-Keplerian rotation is forced on the magnetosphere, the (sub-Keplerian) corotation radius is shifted inward and the position of the disk inner edge with respect to the new corotation radius is relocated. (In principle, the inner edge of the disk could end up beyond the new corotation radius but this would not account for the observed properties of the torque reversal systems.) As a result, the magnetic torque changes and there could be a visible change of spin-up/down torque on the disk transition time scale.

If such a transition does occur at a certain critical rate, the most likely time scales are the local thermal time scale $t_{t h} \sim\left(\alpha \Omega_{K}\right)^{-1}$ or the disk viscous-thermal time scale 
$t_{d i s} \sim R /\left(\alpha c_{s}\right) \sim(R / H)\left(\alpha \Omega_{K}\right)^{-1} \sim 10^{3} s$ for $\alpha \sim 0.3, R \sim 10^{9} \mathrm{~cm}$, and $\dot{M} \sim 10^{16} \mathrm{~g} \mathrm{~s}^{-1}$ (e.g. Frank et al. 1992). This is plausible if the transition is mainly driven by the thermal instability of the local optically thin region of the accretion flow accompanied by the disk density change on the local viscous-thermal time scale (cf. Meyer \& Meyer-Hofmeister 1994). In fact, the viscous time scale for the thin disk is unrealistically long, since the relevant infall time is the one for the hot, and thick disk, which will be only a few orbital times. In any case, these time scales indicate that the local disk surface density change could occur on a time scale short enough, much less than a day, to make the transition appear almost instantaneous. The long term gradual $\dot{M}$ modulation determines the overall evolutionary trend and possibly affects the residuals seen in some observations (Cutler et al. 1986, Chakrabarty 1995). The sudden torque reversal does not require any short term $(\sim d a y)$ change of $\dot{M}$ but only that the condition $\dot{M} \sim \dot{M}_{\text {crit }}$ be satisfied around the time of reversal.

In order to model the reversal episode, we assume no dynamic vertical motion of the disk gas such as winds or outflows. The dominant effect of the transition is to reset the corotation radius and disk truncation radius $R_{o}$. We take the temperature of the sub-Keplerian hot disk to be a constant fraction $\xi$ of the local virial temperature, i.e. $c_{s}^{2} \sim \xi R^{2} \Omega_{K}^{2}$ (e.g. Narayan \& Yi 1995). Then the ratio of the disk thickness to radius is $H / R \sim \xi^{1 / 2}$ and the radial drift velocity is $v_{R} \sim-\alpha \xi R \Omega_{K}$. Using the radial component of the momentum equation (e.g. Campbell 1992), we get the sub-Keplerian rotation frequency $\Omega / \Omega_{K} \sim\left(1-5 \xi / 2-\alpha^{2} \xi^{2} / 2\right)^{1 / 2} \equiv A$. where $\xi \rightarrow 0$ corresponds to the usual Keplerian limit. We note that $v_{R} / R \Omega_{K} \sim \alpha \xi<1$ and $H / R \sim \sqrt{\xi} \leq 1$. Assuming a constant $\xi$ or $A$, after the transition to the sub-Keplerian rotation with $\Omega(R)=A \Omega_{K}(R)<\Omega_{K}(R)$, the corotation radius becomes $R_{c}^{\prime}=A^{2 / 3} R_{c}$ and the new inner disk edge is relocated to $R_{o}^{\prime}$ determined by

$$
\left(\frac{R_{o}^{\prime}}{R_{c}^{\prime}}\right)^{3}=\frac{2 N_{c}}{N_{0}^{\prime} A}\left[1-\left(\frac{R_{o}^{\prime}}{R_{c}^{\prime}}\right)^{3 / 2}\right]
$$

where $N_{0}^{\prime}=A \dot{M}\left(G M_{*} R_{o}^{\prime}\right)^{1 / 2}$. The torque on the star after the transition is

$$
\frac{N^{\prime}}{N_{0}^{\prime}}=\frac{7}{6} \frac{1-(8 / 7)\left(R_{o}^{\prime} / R_{c}^{\prime}\right)^{3 / 2}}{1-\left(R_{o}^{\prime} / R_{c}^{\prime}\right)^{3 / 2}}
$$

The torque vanishes when $R_{o}^{\prime} / R_{c}^{\prime} \rightarrow x_{e q}=0.915$ as in the Keplerian rotation (eq. 2-3). In our discussions, we take a constant $A=0.2$ (e.g. Narayan \& Yi 1995). The parameters $\alpha=0.3$ and $\gamma=1$ are assumed to be constant before and after the transition. For the Keplerian disk, the equilibrium spin $(N=0$ in eq. $(2-3))$ period is

$$
P_{e q}=[4.9 s]\left(\frac{\gamma}{\alpha}\right)^{3 / 7}\left(\frac{B_{*}}{10^{12} G}\right)^{6 / 7}\left(\frac{R_{*}}{10^{6} \mathrm{~cm}}\right)^{18 / 7}\left(\frac{M_{*}}{1.4 M_{\odot}}\right)^{-5 / 7}\left(\frac{\dot{M}}{10^{16} \mathrm{~g} / \mathrm{s}}\right)^{-3 / 7} .
$$

For $\Omega=A \Omega_{K}<\Omega_{K}$, the equilibrium spin period would become longer by a factor $1 / A$ and the system begins to evolve toward the newly determined equilibrium after transition. 


\section{Sudden Torque Reversals}

For the spin evolution calculation we integrate the torque equation

$$
\frac{d P_{*}}{d t}=-\frac{P_{*}^{2}}{I_{*}}[\text { Torque }]
$$

where Torque $=N$ or $N^{\prime}$ depending on the physical state of the inner disk. We take a linear increase or decrease of $\dot{M}$ as an approximation to more complex $\dot{M}$ variations on longer time scales. The transition occurs on a time scale $\ll P_{*} /\left|d P_{*} / d t\right|$ before and after the transition. The transition at $\dot{M}_{c r i t}=10^{15}-10^{16} \mathrm{~g} / \mathrm{s}$, which is determined by the fits to the observed spin evolution, is taken to be instantaneous $\left(\mathrm{cf} . t_{t h}, t_{d i s}\right)$. For each torque reversal event, we adjust

$B_{*}, \dot{M}$, and the accretion rate time scale, $\dot{M} /|d \dot{M} / d t|$. We consider three X-ray pulsars for which abrupt torque reversals have been detected. For a given initial spin period $P_{*}$, a fit gives a set of the above parameters.

4U 1626-67: $4 \mathrm{U} 1626-67\left(P_{*} \approx 7.7 s\right)$ was steadily spun-up on a time scale $\sim 10^{4} \mathrm{yr}$ $\left(\dot{P}_{*} / P_{*}^{2}=-8.54(7) \times 10^{-13} s^{-2}\right)$ during 1979-1989. The Keplerian corotation radius $R_{c} \approx\left(G M_{*} P_{*}^{2} / 4 \pi^{2}\right)^{1 / 3}=6.5 \times 10^{8} \mathrm{~cm}$. The recent BATSE detection of a sudden torque reversal to spin-down is puzzling due to its very short time scale and the nearly equal spinup/down rates. The steady spin-down torque suggests that there remains a dynamically stable (disk) structure after the sudden reversal (For details of observations, see Chakrabarty 1995). In Fig. 1(b), the observed torque reversal event is reproduced by $B_{*}=1.2 \times 10^{11} \mathrm{G}, \dot{M}=4 \times 10^{15} \mathrm{~g} / \mathrm{s}$, and $d \dot{M} / d t=-5 \times 10^{13} \mathrm{~g} / \mathrm{s} / \mathrm{yr}$ which give $R_{o} / R_{c}=0.58, R_{o}^{\prime} / R_{c}^{\prime}=0.95$, and $R_{o}^{\prime} / R_{o}=0.56$. The derived accretion rate is slightly lower than the previously quoted values (Chakrabarty 1995), and accordingly the derived $B_{*}$ is also lower than the previous estimates (e.g. Pravado et al. 1979, Kii et al. 1986, Chakrabarty 1995). We note, however, that our estimated values ( $B_{*}$ and $\dot{M})$ can always be rescaled by changing $\alpha$ and $\gamma$ (Kenyon et al. 1996). The values of $\alpha$ and $\gamma$ based on first principles are not available. The gradual decrease of $\dot{M}$ is consistent with the observed flux decrease (Mavromatakis 1994). The fit naturally achieves the spin-down torque which is slightly smaller than the spin-up torque. The fit requires for the transition to occur at $\dot{M}_{c r i t}=3.3 \times 10^{15} \mathrm{~g} \mathrm{~s}^{-1}$. The gradual decrease of the mass accretion rate on a time scale $\sim 20 y r s$ cannot be due to any viscous or thermal processes operating in the inner region $\left(t_{t h}, t_{d i s}\right)$.

OAO 1657-415: OAO 1657-415 has an observed pulse period $P_{*} \approx 38 s$. Recent observed spin-up/down torques are $\dot{P}_{*} / P_{*}^{2} \approx-7 \times 10^{-12} s^{-2}$ and $\approx 2 \times 10^{-12} s^{-2}$ respectively (Chakrabarty et al. 1993). For the torque reversal in Fig. $1(\mathrm{c})$, we get $B_{*}=10^{12} \mathrm{G}, \dot{M}=2.0 \times 10^{16} \mathrm{~g} / \mathrm{s}$, and $d \dot{M} / d t=-5 \times 10^{16} \mathrm{~g} / \mathrm{s} / \mathrm{yr}$. The characteristic $\dot{M}$ modulation time scale is $\sim 0.3 \mathrm{yr}$. The critical accretion rate $\dot{M}_{c r i t}=1.1 \times 10^{16} \mathrm{~g} / \mathrm{s}$ which is somewhat higher than the value required for $4 \mathrm{U}$ 1626-67.

GX 1+4: GX 1+4 has recently shown a sudden transition from spin-down to spin-up around the spin period $P_{*} \approx 122 s$ (Chakrabarty 1995 and references therein). GX1+4 is peculiar 
in the sense that despite its very short spin time scale $\sim 40 y r$, the spin equilibrium has not been reached. It is likely that $\dot{M}$ fluctuates or oscillates on a time scale $\ll 40 \mathrm{yr}$ near spin equilibrium. The spin-down rate in the 1980's, $\dot{P}_{*} / P_{*}^{2} \sim 3.7 \times 10^{-12} s^{-2}$, is not far from the 1970's spin-up rate, $\dot{P}_{*} / P_{*}^{2} \sim-6.0 \times 10^{-12} s^{-2}$. The recent 1994 torque reversal from spin-down to spin-up lasted for $\sim 100 \mathrm{~d}$. This system also showed very similar spin-down and spin-up rates. Although there is no significant spectral change in the hard X-ray emission spectra during the spin evolution, the flux appears to be increasing as spin-down torque increases (Chakrabarty 1995), which is in contradiction to the behavior expected in the Ghosh-Lamb type model (eqs. $(2-2),(2-3))$. The fit shown in Fig. $1(\mathrm{~d})$ corresponds to $B_{*}=3.2 \times 10^{12} \mathrm{G}, \dot{M}=5.0 \times 10^{15} \mathrm{~g} / \mathrm{s}$, and $d \dot{M} / d t=1.0 \times 10^{16} \mathrm{~g} / \mathrm{s} / \mathrm{yr}$. Once again, we note that these parameters could be rescaled by different choices of $\alpha$ and $\gamma$. It is interesting to observe that the $\dot{M}$ modulation time scale $\dot{M} /(\dot{M} / d t) \sim 5 y r$ is not far from the detected time scale in the coherent variation of pulse frequency residual (Cutler et al. 1986, Chakrabarty 1995). The critical mass accretion rate $\dot{M}_{c r i t}=6.5 \times 10^{15} \mathrm{~g} / \mathrm{s}$ lies between the two values derived above. The gradual decrease of the spin-up torque after reversal is not accurately fit in Fig. 1(d) with the linear $\dot{M}$ variation. This is not surprising given the reported unsteady behavior of the spin-down torque before the reversal (Chakrabarty 1995). The observed $\sim 300 d$ coherent variation in pulse frequency residual is intriguing but such a time scale is not far from the estimated orbital time scale $\sim$ yr (Chakrabarty 1995).

\section{Summary and Discussions}

The proposed transition is most likely to occur at a critical accretion rate $\dot{M}_{c r i t} \sim 10^{15}-10^{16} \mathrm{~g} / \mathrm{s}$. This suggests an interesting connection between the pulsar systems and other compact accretion systems such as cataclysmic variables and black hole soft X-ray transients. We speculate that the transitions seen in these systems may be due to a common physical mechanism, i.e. disk transition to optically thin hot flow. The model indicates that the sudden torque reversal could be a signature of a pulsar system near spin-equilibrium with $\dot{M} \sim \dot{M}_{\text {crit }}$.

There are some outstanding issues to look into. (i) The origin of the gradual $\dot{M}$ modulation on a time scale ranging from $\sim y r$ to a few decades remains unknown. The modulation by the orbital motion on a time scale $\sim y r$ is plausible in GX 1+4 (Chakrabarty 1995) but is unlikely in 4U 1626-67 (Rappaport et al. 1977, Joss et al. 1978, Shinoda et al. 1990, Chakrabarty 1995) and OAO 1657-415 (Chakrabarty et al. 1993). Several binary precession time scales (e.g. Thorne et al. 1986) and the mass flow oscillation time scale (induced by X-ray irradiation, Meyer \& Meyer-Hofmeister 1990) could be relevant for long time scale ( $>$ month) modulations. In $4 \mathrm{U}$ 1626-67 the observed optical and X-ray pulsation frequencies are identical, which has been attributed to the reprocessing of X-rays by accretion disk (Ilovaisky et al. 1978, Chester 1979). 
The direct X-ray irradiation of the secondary star (Hameury et al. 1986) and the disk instability (e.g. Smak 1984) may also give rise to $\dot{M}$ modulations in neutron star systems. (ii) It is important

to quantitatively understand $\dot{M}_{\text {crit }}$ and $R_{\text {crit }}$ (cf. Narayan \& Yi 1995). (iii) Within our model, the observed smooth transitions back to spin-up (seen in OAO 1657-415 and GX 1+4) could result from the return from the advective to Keplerian flow. The characteristic time scale for such a back transition is likely to be the the viscous disk formation time scale. The observed UV-delay time scale, on the order of a day, in cataclysmic variables (Livio \& Pringle 1992), may be similar to the postulated reverse transition time scale. (iv) It remains unexplained that in GX $1+4$ the X-ray flux increased during the increase of the spin-down torque (Chakrabarty 1995). If X-rays come from the shocked polar accretion, as $\dot{M}$ decreases, the X-ray emission temperature and the apparent flux in a fixed X-ray band could decrease due to radiation drag (e.g. Yi \& Vishniac 1994). A geometrically thick, but optically thin, inner disk is more apt to scatter X-ray emission, so that a partially obscured polar cap can actually become more conspicuous as $\dot{M}$ drops.

This research was supported in part by NSF Grant 95-28110 (JCW), by the SUAM Foundation (IY), and by NASA grant NAG5-2773 (ETV). We are happy to acknowledge related discussions on pulsars with Josh Grindlay and Ramesh Narayan.

\section{REFERENCES}

Anzer, U. \& Börner, G. 1995, A\&A, 299, 62

Campbell, C. G. 1992, Geophys. Astrophys. Fluid Dyn., 63, 179

Chakrabarty, D. 1995, Ph.D. Thesis, California Institute of Technology

Chakrabarty, D. et al. 1993, ApJ, 403, L33

Chester, T. J. 1979, ApJ, 227, 569

Cutler, E. P., Dennis, B. R., \& Dolan, J. F. 1986, ApJ, 300, 551

Frank, J., King, A. R., \& Raine, D. 1992, Accretion Power in Astrophysics (Cambridge: Cambridge University Press)

Ghosh, P. \& Lamb, F. K. 1979a, ApJ, 232, 259

Ghosh, P. \& Lamb, F. K. 1979b, 234, 296

Hameury, J.-M., King, A. R., \& Lasota, J.-P. 1986, A\&A, 162, 71

Ilovaisky, S. A., Motch, C., \& Chevalier, C. 1978, A\&A, 70, L19

Joss, P. C., Avni, Y., \& Rappaport, S. 1978, ApJ, 221, 645

Kenyon, S. J., Yi, I., \& Hartmann, L. 1996, ApJ, 462, 439

Kii, T., Hayakawa, S., Nagase, F., Ikegami, T., \& Kawai, N. 1986, PASJ, 38, 751 
Lipunov, V. M. 1992, Astrophysics of Neutron Stars (Berlin: Springer-Verlag)

Livio, M. \& Pringle, J. E. 1992, MNRAS, 259, 23

Mavromatakis, F. 1994, A\&A, 285, 503

Meyer, F. \& Meyer-Hofmeister, E. 1990, A\&A, 239, 214

Meyer, F. \& Meyer-Hofmeister, E. 1994, A\&A, 288, 175

Nagase, F. 1989, PASJ, 41, 1

Narayan, R. \& Popham, R. 1993, Nature, 362, 820

Narayan, R. \& Yi, I. 1995, ApJ, 452, 710

Paczynski, B. 1991, 370, 597

Patterson, J. \& Raymond, J. C. 1985, ApJ, 292, 535

Pravado, S. H. et al. 1979, ApJ, 231, 912

Rappaport, S. et al. 1977, ApJ, 217, L29

Shinoda, K. et al. 1990, PASJ, 42, L27

Smak, J. 1984, PASP, 96, 5

Thorne, K. S., Price, R. H., \& MacDonald, D. A. 1986, Black Holes: The Membrane Paradigm (New Haven: Yale University Press)

Wang, Y.-M. 1995, ApJ, 449, L153

Yi, I. 1995, ApJ, 442, 768

Yi, I. \& Vishniac, E. T. 1994, ApJ, 435, 829 


\section{Figure Caption}

Figure 1: (a) A typical smooth torque reversal event expected in the Ghosh-Lamb type model. This illustrative example assumes $B_{*}=10^{12} G, P_{*}=7.68 \mathrm{~s}$ and $\dot{M}$ linearly decreasing from $7.2 \times 10^{16} \mathrm{~g} / \mathrm{s}$ to $5.0 \times 10^{16} \mathrm{~g} / \mathrm{s}$ over $10 \mathrm{yrs}$. The torque passes through $N=0$ (eq. (2-3)) and the continuous variation should show a wide range of torques before and after the torque reversal. Upper panel: time variation of torque. Lower panel: spin-up to spin-down transition. Examples of (b) 4U 1626-67, (c) OAO 1657-415, and (d) GX 1+4. The solid lines correspond to models described in the text and the dashed lines connect data points adopted from Chakrabarty et al. (1993) and Chakrabarty (1995). In OAO 1657-415 and 4U 1626-67, the observed spin periods are shown only schematically. 


\section{ERRATUM}

In the Letter "Torque Reversal in Accretion-Powered X-ray Pulsars" by I. Yi, J. C. Wheeler, \& E. T. Vishniac (ApJ, 481, L51 [1997]), there are errors in the values of the magnetic fields and the mass accretion rates. The correct values are as follows and Figure 1 is revised. 4U 1626-67: $B_{*}=6 \times 10^{11} G, \dot{M}=2.8 \times 10^{16} \mathrm{~g} / \mathrm{s}, d \dot{M} / d t=-4.5 \times 10^{14} \mathrm{~g} / \mathrm{s} / \mathrm{yr}, R_{o} / R_{c}=0.760, R_{o}^{\prime} / R_{c}^{\prime}=0.987$, $R_{o}^{\prime} / R_{o}=0.444, \dot{M}_{c r i t}=2.2 \times 10^{16} \mathrm{~g} / \mathrm{s}$. OAO 1657-415: $B_{*}=5 \times 10^{12} \mathrm{G}, \dot{M}=1.2 \times 10^{17} \mathrm{~g} / \mathrm{s}$, $d \dot{M} / d t=-1.2 \times 10^{17} \mathrm{~g} / \mathrm{s} / \mathrm{yr}, \dot{M}_{\text {crit }}=1.0 \times 10^{17} \mathrm{~g} / \mathrm{s}$. GX 1+4: $B_{*}=1.3 \times 10^{13} \mathrm{G}, \dot{M}=4 \times 10^{16} \mathrm{~g} / \mathrm{s}$, $d \dot{M} / d t=1.3 \times 10^{17} \mathrm{~g} / \mathrm{s} / \mathrm{yr}, \dot{M}_{c r i t}=6 \times 10^{16} \mathrm{~g} / \mathrm{s}$.

\section{Figure 1-Corrected}

Torque reversal events in three X-ray pulsar systems. Short dashed lines correspond to the smooth torque transition (see text) and thick solid lines correspond to the sudden torque transition proposed in the present work. The points connected by long dashed lines are observed data points adopted from Chakrabarty et al. (1993) and Chakrabarty (1995). In OAO 1657-415 and $4 \mathrm{U}$ 1626-67, the observed spin periods are shown only schematically. Examples of (a) $4 \mathrm{U}$ 1626-67, (b) OAO 1657-415, and (c) GX 1+4. For the smooth transition events, the parameters $\left(B_{*}, \dot{M}, d \dot{M} / d t\right)$ are $\left(10^{12} \mathrm{G}, 4.2 \times 10^{16} \mathrm{~g} / \mathrm{s},-2 \times 10^{15} \mathrm{~g} / \mathrm{s} / \mathrm{yr}\right)$ for $4 \mathrm{U} 1626-67,\left(10^{13} \mathrm{G}, 2 \times 10^{17} \mathrm{~g} / \mathrm{s}\right.$, $\left.-5 \times 10^{17} \mathrm{~g} / \mathrm{s} / \mathrm{yr}\right)$ for OAO $1657-415$, and $\left(4 \times 10^{13} \mathrm{G}, 10^{15} \mathrm{~g} / \mathrm{s}, 1.8 \times 10^{17} \mathrm{~g} / \mathrm{s} / \mathrm{gr}\right)$ for GX $1+4$. 

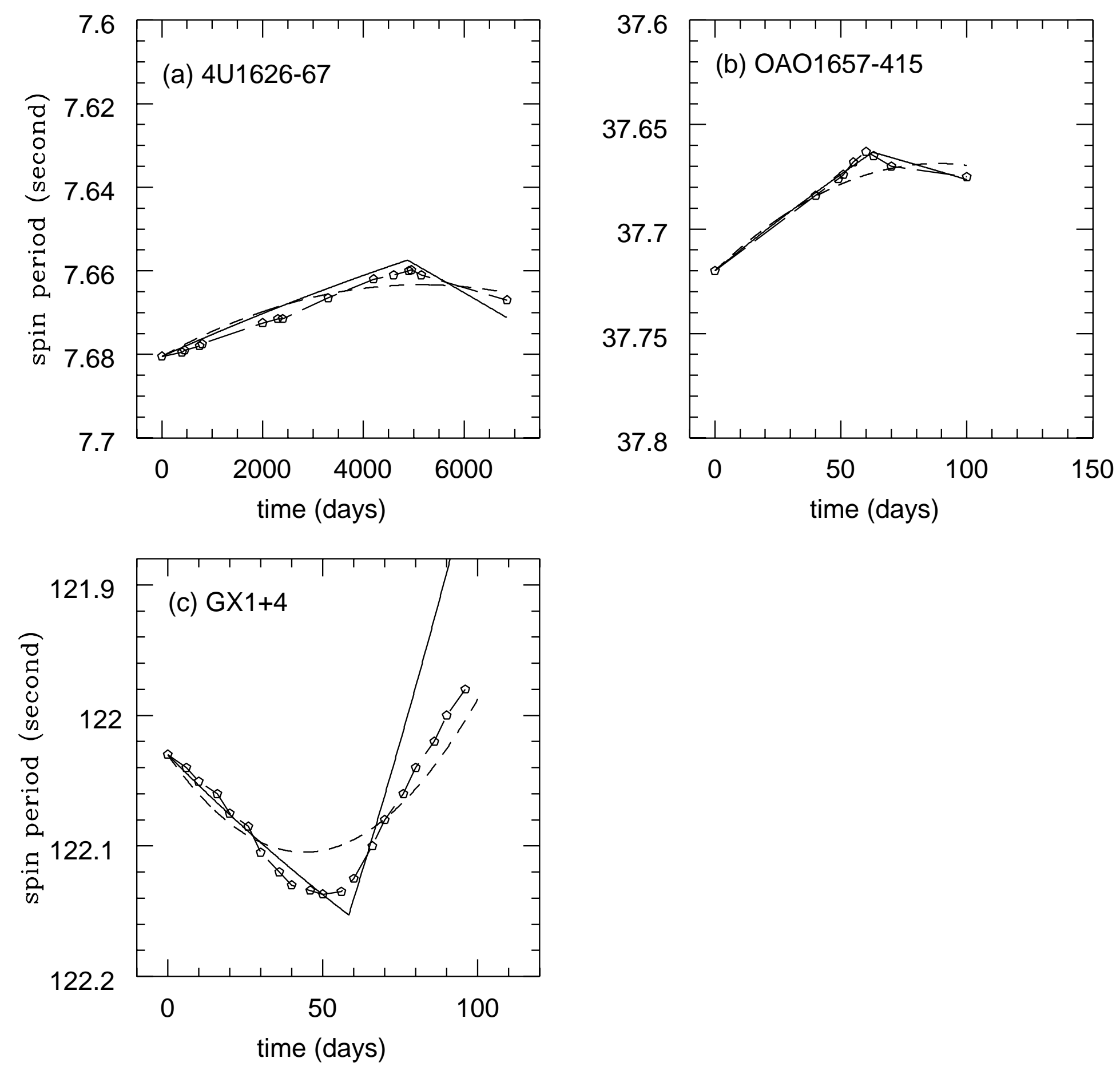